\title{
Certain New Schläfli Type Mixed Modular Equations
}

\author{
Megadahalli S. Mahadeva Naika ${ }^{1}$. \\ Sathyanarayana Chandankumar ${ }^{2}$. Manjunath Harish ${ }^{1}$
}

Received: 20 May 2014 / Accepted: 21 March 2015

(C) Vietnam Academy of Science and Technology (VAST) and Springer Science+Business Media Singapore 2015

\begin{abstract}
Schläfli (J. Reine Angew. Math. 72, 360-369, 1870) has established modular equations involving $k k^{\prime}$ and $\lambda \lambda^{\prime}$ for degrees 3, 5, 7, 9, 11, 13, 17, and 19. On pages 86 and 88 of his first notebook, Ramanujan recorded 11 Schläfli-type modular equations for composite degrees. In this paper, we establish several new Schläfli type mixed modular equations for composite degrees by elementary algebraic manipulations which are analogous to those recorded by Ramanujan.
\end{abstract}

Keywords Modular equations · Theta-functions

Mathematics Subject Classification (2010) 33D10 - 11A55 - 11F27

\section{Introduction}

Following Ramanujan, let $(a ; q)_{\infty}$ denote the infinite product $\prod_{n=0}^{\infty}\left(1-a q^{n}\right)(a, q$ are complex numbers, $|q|<1)$ and the Ramanujan theta-function $f(a, b)$ :

$$
f(a, b):=\sum_{n=-\infty}^{\infty} a^{n(n+1) / 2} b^{n(n-1) / 2}, \quad|a b|<1,
$$

Megadahalli S. Mahadeva Naika

msmnaika@rediffmail.com

Sathyanarayana Chandankumar

chandan.s17@gmail.com

Manjunath Harish

numharish@gmail.com

1 Department of Mathematics, Bangalore University, Central College Campus,

Bangalore, 560001 Karnataka, India

2 Department of Mathematics, M. S. Ramaiah University of Applied Sciences, Peenya Campus,

Peenya 4th Phase, Bengaluru, 560058 Karnataka, India 
related to the above product by Jacobi's fundamental factorization formula

$$
f(a, b)=(-a ; a b)_{\infty}(-b ; a b)_{\infty}(a b ; a b)_{\infty} .
$$

The following definitions of theta-functions $\varphi$ with $|q|<1$ are classical:

$$
\begin{gathered}
\varphi(q):=f(q, q)=\sum_{n=-\infty}^{\infty} q^{n^{2}}=\left(-q ; q^{2}\right)_{\infty}^{2}\left(q^{2} ; q^{2}\right)_{\infty}, \\
f(-q):=f\left(-q,-q^{2}\right)=\sum_{n=-\infty}^{\infty}(-1)^{n} q^{n(3 n-1) / 2}=(q ; q)_{\infty} .
\end{gathered}
$$

The ordinary or Gaussian hypergeometric function is defined by

$$
{ }_{2} F_{1}(a, b ; c ; z):=\sum_{n=0}^{\infty} \frac{(a)_{n}(b)_{n}}{(c)_{n} n !} z^{n}, \quad 0 \leq|z|<1,
$$

where $a, b$, and $c$ are complex numbers such that $c \neq 0,-1,-2, \ldots$, and

$$
(a)_{0}=1, \quad(a)_{n}=a(a+1) \cdots(a+n-1) \text { for any positive integer } n .
$$

Now, we recall the notion of modular equation. Let $K(k)$ be the complete elliptic integral of the first kind of modulus $k$. Recall that

$$
K(k):=\int_{0}^{\frac{\pi}{2}} \frac{d \phi}{\sqrt{1-k^{2} \sin ^{2} \phi}}=\frac{\pi}{2} \sum_{n=0}^{\infty} \frac{\left(\frac{1}{2}\right)^{2}}{(n !)^{2}} k^{2 n}=\frac{\pi}{2}{ }_{2} F_{1}\left(\frac{1}{2}, \frac{1}{2}, 1, k^{2}\right),
$$

where $0<k<1$. Set $K^{\prime}=K\left(k^{\prime}\right)$, where $k^{\prime}=\sqrt{1-k^{2}}$ is the so called complementary modulus of $k$. It is classical to set $q(k)=e^{-\pi K\left(k^{\prime}\right) / K(k)}$ so that $q$ is one-to-one increasing from 0 to 1 .

In the same manner introduce $L_{1}=K\left(\ell_{1}\right), L_{1}^{\prime}=K\left(\ell_{1}^{\prime}\right)$ and suppose that the following equality

$$
n_{1} \frac{K^{\prime}}{K}=\frac{L_{1}^{\prime}}{L_{1}}
$$

holds for some positive integer $n_{1}$. Then a modular equation of degree $n_{1}$ is an algebraic relation between the moduli $k$ and $\ell_{1}$ which is induced by (2). Following Ramanujan, we set $\alpha=k^{2}$ and $\beta=\ell_{1}^{2}$, then we say $\beta$ is of degree $n_{1}$ over $\alpha$. The multiplier $m$ is defined by

$$
m=\frac{K}{L_{1}}=\frac{\varphi^{2}(q)}{\varphi^{2}\left(q^{n_{1}}\right)}
$$

for $q=e^{-\pi K\left(k^{\prime}\right) / K(k)}$.

Let $K, K^{\prime}, L_{1}, L_{1}^{\prime}, L_{2}, L_{2}^{\prime}, L_{3}$, and $L_{3}^{\prime}$ denote complete elliptic integrals of the first kind corresponding, in pairs, to the moduli $\sqrt{\alpha}, \sqrt{\beta}, \sqrt{\gamma}$, and $\sqrt{\delta}$, and their complementary moduli, respectively. Let $n_{1}, n_{2}$, and $n_{3}$ be positive integers such that $n_{3}=n_{1} n_{2}$. Suppose that the equalities

$$
n_{1} \frac{K^{\prime}}{K}=\frac{L_{1}^{\prime}}{L_{1}}, \quad n_{2} \frac{K^{\prime}}{K}=\frac{L_{2}^{\prime}}{L_{2}}, \quad \text { and } \quad n_{3} \frac{K^{\prime}}{K}=\frac{L_{3}^{\prime}}{L_{3}}
$$

hold. Then a "mixed" modular equation is a relation between the moduli $\sqrt{\alpha}, \sqrt{\beta}, \sqrt{\gamma}$, and $\sqrt{\delta}$ that is induced by (4). We say that $\beta, \gamma$, and $\delta$ are of degrees $n_{1}, n_{2}$, and $n_{3}$, respectively, over $\alpha$. The multipliers $m=K / L_{1}$ and $m^{\prime}=L_{2} / L_{3}$ are algebraic relation involving $\alpha, \beta$, $\gamma$, and $\delta$. 
Jacobi and Sohncke have established modular equations involving $\alpha^{1 / 8}$ and $\beta^{1 / 8}$. Such modular equations are called Jacobi-Sohncke modular equations.

Russell [15] has obtained several modular equations of degrees $n=13,17,29,43,53$, and 59 involving $(\alpha \beta)^{1 / 8}$ and $\{(1-\alpha)(1-\beta)\}^{1 / 8}$, which are referred to as Russell type modular equations. For example

$$
(\alpha \beta)^{1 / 4}+\{(1-\alpha)(1-\beta)\}^{1 / 4}=1,
$$

where $\beta$ is of degree 3 over $\alpha$.

Schläfli has established modular equations for degrees $3,5,7,9,11,13,17$, and 19 involving $\{4 \alpha(1-\alpha)\}^{1 / 24}$ and $\{4 \beta(1-\beta)\}^{1 / 24}$. These modular equations are known as Schläfli type modular equations. Weber [18, p. 90] has also established irrational modular equation for composite degrees involving $f, f_{1}, f_{2}$, and $f_{3}$, where $f=\{4 \alpha(1-\alpha)\}^{1 / 24}$, $f_{1}=\{4 \beta(1-\beta)\}^{1 / 24}, f_{2}=\{4 \gamma(1-\gamma)\}^{1 / 24}$, and $f_{3}=\{4 \delta(1-\delta)\}^{1 / 24}$, which are known as Weber type modular equations.

Ramanujan's class invariant $G_{n}$ is defined by

$$
G_{n}:=2^{-1 / 4} q^{-1 / 24} \chi(q),
$$

where

$$
\chi(q)=\left(-q ; q^{2}\right)_{\infty}, \quad q=\exp (-\pi \sqrt{n}) .
$$

Since $\chi(q)=2^{1 / 6}\{\alpha(1-\alpha) / q\}^{-1 / 24}$ [3, Ch. 17, Entry 12(v), p. 124], it follows from (5) that

$$
G_{n}=\{4 \alpha(1-\alpha)\}^{-1 / 24} .
$$

Recently, Mahadeva Naika [6] and Mahadeva Naika and Sushan Bairy [10] have obtained several explicit evaluations of class invariants using modular equations.

On pages 86 and 88 of his first notebook [14], Ramanujan recorded a total of 11 Schläfli-type modular equations for composite degrees. In [13], Ramanathan observed that one of these 11 equations follows from a modular equation recorded by Ramanujan in Chapter 20 of second notebook. By using the theory of modular forms, Berndt [4] has proved these modular equations. In [1, 2], Baruah proved these equations by deriving some theta-function identities from Schröter's formulae and using Ramanujan's theta-function identities. Mahadeva Naika and Bairy [11] have also established several new mixed modular equations in the form of Schläfli. For more details one can see [5, 7, 9, 12, 17].

Motivated by all these works, in this paper, we establish nine new mixed modular equations in the form of Schläfli. We utilize some identities involving ratios of theta-function in establishing these equations.

\section{Preliminary Results}

In this section, we list some of the relevant identities which are useful in proving of our results.

Lemma 1 [3, Ch. 17, Entry 12 (i) and (iii), p. 124] For $0<x<1$, we have

$$
\begin{aligned}
f\left(e^{-y}\right) & =\sqrt{z} 2^{-1 / 6}\left\{x(1-x) e^{y}\right\}^{1 / 24}, \\
f\left(e^{-2 y}\right) & =\sqrt{z} 2^{-1 / 3}\left\{x(1-x) e^{y}\right\}^{1 / 12},
\end{aligned}
$$

where $z:={ }_{2} F_{1}\left(\frac{1}{2}, \frac{1}{2} ; 1 ; x\right)$ and $y:=\pi \frac{{ }_{2} F_{1}\left(\frac{1}{2}, \frac{1}{2} ; 1 ; 1-x\right)}{{ }_{2} F_{1}\left(\frac{1}{2}, \frac{1}{2} ; 1 ; x\right)}$. 
Lemma 2 [3, Ch. 19, Entry 13 (xii) and (xiii), pp. 281-282] If $\beta$ is of degree 5 over $\alpha$, then

$$
\begin{aligned}
& \left(\frac{\beta}{\alpha}\right)^{1 / 4}+\left(\frac{1-\beta}{1-\alpha}\right)^{1 / 4}-\left(\frac{\beta(1-\beta)}{\alpha(1-\alpha)}\right)^{1 / 4}=m, \\
& \left(\frac{\alpha}{\beta}\right)^{1 / 4}+\left(\frac{1-\alpha}{1-\beta}\right)^{1 / 4}-\left(\frac{\alpha(1-\alpha)}{\beta(1-\beta)}\right)^{1 / 4}=\frac{5}{m}
\end{aligned}
$$

where $m$ is the multiplier for degree 5.

Lemma 3 [8] If $X:=\frac{\varphi(q)}{\varphi\left(q^{5}\right)}$ and $Y:=\frac{\varphi\left(q^{4}\right)}{\varphi\left(q^{20}\right)}$, then

$$
\begin{aligned}
& {\left[\frac{X^{4}}{Y^{4}}+\frac{Y^{4}}{X^{4}}\right]-112\left[\frac{X^{3}}{Y^{3}}+\frac{Y^{3}}{X^{3}}\right]+1440\left[\frac{X^{2}}{Y^{2}}+\frac{Y^{2}}{X^{2}}\right]-3184\left[\frac{X}{Y}+\frac{Y}{X}\right]} \\
& +\left[X^{4} Y^{4}+\frac{5^{4}}{X^{4} Y^{4}}\right]-\left[X^{3} Y^{3}+\frac{5^{3}}{X^{3} Y^{3}}\right]\left\{4\left[\frac{X}{Y}+\frac{Y}{X}\right]+16\right\} \\
& +\left[X^{2} Y^{2}+\frac{5^{2}}{X^{2} Y^{2}}\right]\left\{48\left[\frac{X}{Y}+\frac{Y}{X}\right]+6\left[\frac{X^{2}}{Y^{2}}+\frac{Y^{2}}{X^{2}}\right]+128\right\} \\
& -\left[X Y+\frac{5}{X Y}\right]\left\{1360-248\left[\frac{X}{Y}+\frac{Y}{X}\right]+176\left[\frac{X^{2}}{Y^{2}}+\frac{Y^{2}}{X^{2}}\right]+4\left[\frac{X^{3}}{Y^{3}}+\frac{Y^{3}}{X^{3}}\right]\right\}+7316=0 .
\end{aligned}
$$

Lemma 4 [8] If $X:=\frac{\varphi(q)}{\varphi\left(q^{5}\right)}$ and $Y:=\frac{\varphi\left(q^{5}\right)}{\varphi\left(q^{25}\right)}$, then

$$
\frac{Y^{3}}{X^{3}}-\frac{5 Y^{2}}{X^{2}}-\frac{15 Y}{X}+5\left[X Y+\frac{5}{X Y}\right]+5\left[Y^{2}+\frac{5}{X^{2}}\right]=X^{2} Y^{2}+\frac{5^{2}}{X^{2} Y^{2}}+15
$$

Lemma 5 [8] If $X:=\frac{\varphi(q) \varphi\left(q^{11}\right)}{\varphi\left(q^{5}\right) \varphi\left(q^{55}\right)}$ and $Y:=\frac{\varphi(q) \varphi\left(q^{55}\right)}{\varphi\left(q^{5}\right) \varphi\left(q^{11}\right)}$, then

$$
\begin{aligned}
& Y^{6}+\frac{1}{Y^{6}}+33\left[Y^{5}+\frac{1}{Y^{5}}\right]-99\left[Y^{4}+\frac{1}{Y^{4}}\right]+1529\left[Y^{3}+\frac{1}{Y^{3}}\right] \\
& -1683\left[Y^{2}+\frac{1}{Y^{2}}\right]+8800\left[Y+\frac{1}{Y}\right]=6534+\left[X^{5}+\frac{5^{5}}{X^{5}}\right] \\
& -11\left\{\left[X^{4}+\frac{5^{4}}{X^{4}}\right]\left[Y+\frac{1}{Y}\right]-\left[X^{3}+\frac{5^{3}}{X^{3}}\right]\left[11+4\left[Y^{2}+\frac{1}{Y^{2}}\right]\right]\right. \\
& -\left[X^{2}+\frac{5^{2}}{X^{2}}\right]\left[18-56\left[Y+\frac{1}{Y}\right]+3\left[Y^{2}+\frac{1}{Y^{2}}\right]-8\left[Y^{3}+\frac{1}{Y^{3}}\right]\right] \\
& \left.-\left[X+\frac{5}{X}\right]\left[-126\left[Y+\frac{1}{Y}\right]+160\left[Y^{2}+\frac{1}{Y^{2}}\right]-18\left[Y^{3}+\frac{1}{Y^{3}}\right]+324+9\left[Y^{4}+\frac{1}{Y^{4}}\right]\right]\right\} .
\end{aligned}
$$

\section{Mixed Modular Equations in the Form of Schläfli}

In this section, we establish several new Schläfli type mixed modular equations. We set

$$
\begin{aligned}
P & :=(256 \alpha \beta \gamma \delta(1-\alpha)(1-\beta)(1-\gamma)(1-\delta))^{1 / 24} \\
Q & :=\left(\frac{\alpha \delta(1-\alpha)(1-\delta)}{\beta \gamma(1-\beta)(1-\gamma)}\right)^{1 / 24} \\
R & :=\left(\frac{\gamma \delta(1-\gamma)(1-\delta)}{\alpha \beta(1-\alpha)(1-\beta)}\right)^{1 / 24} \\
T & :=\left(\frac{\alpha \gamma(1-\alpha)(1-\gamma)}{\beta \delta(1-\beta)(1-\delta)}\right)^{1 / 24}
\end{aligned}
$$


Theorem 1 If $\alpha, \beta, \gamma$, and $\delta$ have degrees 1, 5, 5, and 25, respectively, then

$$
\begin{aligned}
& 16\left(T+\frac{1}{T}\right)+11\left(T^{2}+\frac{1}{T^{2}}\right)+6\left(T^{3}+\frac{1}{T^{3}}\right)+\left(T^{4}+\frac{1}{T^{4}}\right)+22 \\
& =\left(Q^{3}+\frac{1}{Q^{3}}\right)\left[\left(T+\frac{1}{T}\right)+\left(T^{2}+\frac{1}{T^{2}}\right)+1\right] .
\end{aligned}
$$

Proof Equations (9) and (10) can be rewritten as

$$
m+\left\{\frac{\beta(1-\beta)}{\alpha(1-\alpha)}\right\}^{1 / 4}=\frac{5}{m}\left\{\frac{\beta(1-\beta)}{\alpha(1-\alpha)}\right\}^{1 / 4}+1 .
$$

Equation (19) can be written as

$$
m+a=\frac{5 a}{m}+1
$$

where $m:=\frac{\varphi^{2}(q)}{\varphi^{2}\left(q^{5}\right)}$ and $a:=\left\{\frac{\beta(1-\beta)}{\alpha(1-\alpha)}\right\}^{1 / 4}$.

In a similar manner, we obtain

$$
m^{\prime}+b=\frac{5 b}{m^{\prime}}+1,
$$

where $m^{\prime}:=\frac{\varphi^{2}\left(q^{5}\right)}{\varphi^{2}\left(q^{25}\right)}$ and $b:=\left\{\frac{\delta(1-\delta)}{\gamma(1-\gamma)}\right\}^{1 / 4}$.

Employing (20) and (21) in (12), we deduce that

$$
\begin{aligned}
& 71 X-5 Y a u b+5 Y a u v-13 X b v a+4 X b v u-8 X a u b-X a u v-X a^{2} b v \\
& -X a u b^{2}+X a u b v+10 Y v u+2 Y b v-6 X b v-55 Y v a+55 Y b a-10 Y b u \\
& -42 Y-26 Y b+8 Y v-2 Y b^{2}+83 X b-29 X v+6 X b^{2}-40 Y u-5 Y a^{2} \\
& -2 X a+11 X u+X a^{2}-5 Y a+5 Y a^{2} b-5 Y a^{2} v+5 Y a u+119 X b a-47 X b u \\
& -2 X v a+11 X v u+13 X b^{2} a-4 X b^{2} u-X a u+8 X a^{2} b+X a^{2} v+X a^{2} b^{2}=0,
\end{aligned}
$$

where $u=\sqrt{(a-1)^{2}+20 a}$ and $v=\sqrt{(b-1)^{2}+20 b}$.

Collecting the terms containing $X$ on one side of (22) and then squaring both sides, we find that

$$
\begin{aligned}
& 624-106 a u b^{3} v-827 a^{2} u b^{2} v-21 a^{3} u b^{3} v+132 a^{3} u b v-152 a^{3} u b^{2} v \\
& +462 a^{2} u b v-1032 a u b^{2} v-453 a u b v-106 a^{2} u b^{3} v-a^{4} u b^{3} v+7 a^{4} u b v \\
& -7 a^{4} u b^{2} v-650 a+255 b^{3} a^{3} v+106 b^{4} a^{2} u+16 a^{4} u b^{2}+16 a^{4} u b+16 a^{4} u b^{3} \\
& +a^{4} u v+a^{4} b^{4} u+a^{5} b^{3} v-7 a^{5} b v+7 a^{5} b^{2} v-195 a^{4} b v+1915 b^{2} a^{3} v \\
& +215 a^{4} b^{2} v+255 b^{3} v a-21 b^{3} v u+580 b^{3} a^{2} v+106 b^{4} a u-1370 a^{3} b v \\
& +5501 a u b^{2}-945 a^{2} b v-1013 b v u+16 a u v+4330 b v a+941 a u b \\
& +4915 a^{2} b^{2} v+16 a^{3} u v+1781 a^{2} u b^{3}+276 a^{3} u b+2965 b^{2} v a+2741 a^{2} u b^{2} \\
& +396 a^{3} u b^{2}+46 a^{2} u v+876 a^{2} u b-317 b^{2} v u+341 a^{3} u b^{3}+30 a^{4} b^{3} v \\
& +2906 b^{2}+626 u+4581 b-626 v-150 a^{2}-150 a^{3}-a^{5}-25 a^{4}+626 b^{3} \\
& +61 b^{4}+2 b^{5}-540 a^{4} b^{2}-485 a^{4} b^{3}-255 b^{4} a^{3}-30 a^{4} b^{4}-2 b^{4} v-1035 b v \\
& -12770 b a+4621 b u+600 v a-624 v u+16 a u-3545 a^{2} b-150 a^{2} v \\
& -20815 b^{2} a+3026 b^{2} u-20090 a^{2} b^{2}-16 a^{5} b^{2}-16 a^{5} b^{3}+a^{4} u-a^{5} b^{4} \\
& -a^{5} v+46 a^{2} u-2720 a^{3} b-150 a^{3} v-5665 a^{3} b^{2}-319 b^{2} v+1986 a u b^{3} \\
& -10135 a^{2} b^{3}-43 b^{3} v-255 b^{4} a+21 b^{4} u-580 b^{4} a^{2}-5260 b^{3} a+506 b^{3} u \\
& -4210 b^{3} a^{3}+16 a^{3} u-420 a^{4} b-25 a^{4} v-16 a^{5} b+21 a^{3} b^{4} u=0 .
\end{aligned}
$$


Isolating the terms containing $u$ on one side of (23) and then squaring both sides, we deduce

$$
\begin{aligned}
& 580 b^{8} a^{2} v+603730 a^{2} b^{6} v+95401 b^{6}+16686 b^{7}+1446 b^{8}+61 b^{9}+b^{10} \\
& -1953125 a+15926770 b^{3} a^{3} v+57344 a^{5} b^{3} v-2048 a^{5} b v+7168 a^{5} b^{2} v \\
& +5618575 b^{5} a^{2} v+a^{5} b^{8} v+52 a^{5} b^{7} v+13290 b^{7} v a^{3}+2960950 b^{5} v a \\
& -59520 b^{6} v a^{4}+290005 b^{6} a v+2297700 b^{5} a^{3} v+1304050 a^{4} b^{4} v+255 b^{8} a^{3} v \\
& +30415 a^{2} b^{7} v-143550 b^{5} a^{4} v+1018 b^{6} v a^{5}+9608925 b^{4} a^{3} v-2535 b^{7} v a^{4} \\
& +255 b^{8} a v+257055 a^{3} b^{6} v+9244 b^{5} a^{5} v+24479800 b^{4} a^{2} v+1116160 a^{4} b^{2} v \\
& +15371050 a b^{4} v+38245 a^{5} b^{4} v+261120 a^{4} b v+3402085 b^{2} a^{3} v \\
& +39102520 b^{3} v a+42868445 b^{3} a^{2} v+13840 b^{7} v a+593920 a^{3} b v+30 a^{4} b^{8} v \\
& +19561970 b v a+20084210 a^{2} b^{2} v+44696285 b^{2} v a+1574245 a^{4} b^{3} v \\
& +11264 b^{2}+1024 b-1024 a^{5}-29696 b^{3}+164864 b^{4}+238949 b^{5} \\
& -2731365 a^{4} b^{3}-62291095 b^{4} a^{3}-9389695 a^{4} b^{4}-57344 b^{4} v-16686 b^{6} a^{5} \\
& -4171395 b^{6} a^{3}+567030 b^{6} a^{4}-5109195 b^{6} a-10044345 b^{6} a^{2}-9244 b^{6} v \\
& -23760225 b^{5} a^{3}-1359100 a^{4} b^{5}-38245 b^{5} v-34371600 a b^{5}-95401 a^{5} b^{5} \\
& -59503975 b^{5} a^{2}-404365 b^{7} a-854265 a^{2} b^{7}-1446 a^{5} b^{7}-61 a^{5} b^{8}-a^{5} b^{9} \\
& -366465 a^{3} b^{7}-1018 b^{7} v+83535 b^{7} a^{4}-16135 b^{8} a-35635 a^{2} b^{8}-52 b^{8} v \\
& -15585 b^{8} a^{3}+2265 b^{8} a^{4}-255 b^{9} a-580 b^{9} a^{2}-255 b^{9} a^{3}-30 a^{4} b^{9}-b^{9} v \\
& +1024 b v-37078655 b a+1953125 v a-1692005 a^{2} b-150339735 b^{2} a \\
& -34206335 a^{2} b^{2}+29696 a^{5} b^{2}-164864 a^{5} b^{3}-238949 a^{5} b^{4}-11264 a^{5} b \\
& -1024 a^{5} v+593920 a^{3} b+484115 a^{3} b^{2}+2048 b^{2} v-204390965 b^{3} a \\
& -157912215 a^{2} b^{3}-7168 b^{3} v-120435970 b^{4} a-162350645 b^{4} a^{2} \\
& -51430015 b^{3} a^{3}+261120 a^{4} b+3466240 a^{4} b^{2}+2214245 a^{2} b v=0 \\
& +24
\end{aligned}
$$

Eliminating $v$ from (24), we obtain

$-b a-30 a^{2} b-30 b^{2} a+3194 a^{2} b^{2}-8810 a^{5} b^{2}-100590 a^{5} b^{3}-466770 a^{5} b^{4}$ $-255 a^{5} b-255 a^{3} b-7710 a^{3} b^{2}-255 b^{3} a-7710 a^{2} b^{3}-580 b^{4} a-17910 b^{4} a^{2}$ $-58636 b^{3} a^{3}-580 a^{4} b-17910 a^{4} b^{2}-163290 a^{4} b^{3}-163290 b^{4} a^{3}-456866 a^{4} b^{4}$ $-466770 b^{6} a^{5}-8230 b^{6} a^{3}-100335 b^{6} a^{4}+30 b^{6} a-466770 b^{5} a^{6}-100590 a^{7} b^{5}$ $+30 a^{7} b^{2}-8230 a^{7} b^{4}+a^{7} b-100590 b^{5} a^{3}-466770 a^{4} b^{5}-8230 a^{6} b^{3}$ $-100335 a^{6} b^{4}+30 a^{6} b-255 a b^{5}-920122 a^{5} b^{5}-8810 b^{5} a^{2}+a^{6}+b^{6}$ $-456866 b^{6} a^{6}+b^{7} a-163290 a^{7} b^{6}+30 a^{2} b^{7}+a^{10} b^{4}+30 a^{8} b^{3}-8810 a^{8} b^{5}$ $+a^{9} b^{3}+30 a^{9} b^{4}-255 a^{9} b^{5}-17910 a^{8} b^{6}-580 a^{9} b^{6}+a^{8} b^{2}-100590 a^{5} b^{7}$ $-163290 a^{6} b^{7}-58636 a^{7} b^{7}-7710 a^{8} b^{7}-255 a^{9} b^{7}-8810 a^{5} b^{8}-17910 a^{6} b^{8}$ $-7710 a^{7} b^{8}+3194 a^{8} b^{8}-30 a^{9} b^{8}-255 a^{5} b^{9}-8230 b^{7} a^{4}+a^{2} b^{8}+30 b^{8} a^{3}$ $+b^{9} a^{3}+30 a^{4} b^{9}-580 a^{6} b^{9}+a^{4} b^{10}-255 a^{7} b^{9}-30 a^{8} b^{9}-a^{9} b^{9}=0$. 
Simplifying the above equation (25) after substituting for $a$ and $b$, we deduce that

$$
\begin{aligned}
& \left(-Q^{6}+16 Q^{3} T^{3}+T^{3}-Q^{3}-Q^{4}-Q^{2}-Q^{5}+6 Q^{7} T^{3}+16 Q^{5} T^{3}-Q^{6} T^{6}\right. \\
& -Q^{4} T^{6}-Q^{2} T^{6}+Q^{8} T^{3}-Q^{5} T^{6}-Q^{3} T^{6}+11 Q^{6} T^{3}+22 Q^{4} T^{3}+11 Q^{2} T^{3} \\
& \left.+6 T^{3} Q\right)\left(Q^{12}+5 Q^{12} T^{6}-10 Q^{9} T^{3}+4 Q^{3} T^{3}+Q^{12} T^{12}+Q^{9} T^{12}+Q^{9}\right. \\
& +T^{6}-10 Q^{9} T^{9}+70 Q^{11} T^{6}+Q^{14} T^{3}+Q^{14} T^{9}-Q^{11} T^{12}+10 Q^{8} T^{9} \\
& -Q^{5} T^{12}-70 Q^{9} T^{6}-15 Q^{12} T^{3}-15 Q^{12} T^{9}+Q^{4}-Q^{11}-Q^{5}-Q^{8}+Q^{7} \\
& -10 Q^{7} T^{3}+16 Q^{5} T^{3}+16 Q^{11} T^{3}+176 Q^{8} T^{6}+25 Q^{14} T^{6}+4 Q^{13} T^{3} \\
& -60 Q^{10} T^{6}-60 Q^{6} T^{6}+5 Q^{4} T^{6}-10 Q^{7} T^{9}+16 Q^{5} T^{9}+16 Q^{11} T^{9}-Q^{8} T^{12} \\
& +4 Q^{13} T^{9}+Q^{16} T^{6}+25 Q^{2} T^{6}+4 Q^{3} T^{9}+Q^{4} T^{12}+5 Q^{10} T^{3}-70 Q^{7} T^{6} \\
& +10 Q^{8} T^{3}-34 Q^{13} T^{6}+70 Q^{5} T^{6}-34 Q^{3} T^{6}+5 Q^{6} T^{3}-15 Q^{4} T^{3}-15 Q^{4} T^{9} \\
& \left.+5 Q^{10} T^{9}+Q^{7} T^{12}-6 Q^{15} T^{6}-6 Q T^{6}+Q^{2} T^{3}+Q^{2} T^{9}+5 Q^{6} T^{9}\right) \\
& \left(Q^{12}+5 Q^{12} T^{6}-10 Q^{9} T^{3}+4 Q^{3} T^{3}+Q^{12} T^{12}-Q^{9} T^{12}-Q^{9}+T^{6}\right. \\
& -10 Q^{9} T^{9}-70 Q^{11} T^{6}-Q^{14} T^{3}-Q^{14} T^{9}+Q^{11} T^{12}-10 Q^{8} T^{9}+Q^{5} T^{12} \\
& +70 Q^{9} T^{6}+15 Q^{12} T^{3}+15 Q^{12} T^{9}+Q^{4}+Q^{11}+Q^{5}-Q^{8}-Q^{7}-10 Q^{7} T^{3} \\
& +16 Q^{5} T^{3}+16 Q^{11} T^{3}+176 Q^{8} T^{6}+25 Q^{14} T^{6}+4 Q^{13} T^{3}-60 Q^{10} T^{6} \\
& -60 Q^{6} T^{6}+5 Q^{4} T^{6}-10 Q^{7} T^{9}+16 Q^{5} T^{9}+16 Q^{11} T^{9}-Q^{8} T^{12}+4 Q^{13} T^{9} \\
& +Q^{16} T^{6}+25 Q^{2} T^{6}+4 Q^{3} T^{9}+Q^{4} T^{12}-5 Q^{10} T^{3}+70 Q^{7} T^{6}-10 Q^{8} T^{3} \\
& +34 Q^{13} T^{6}-70 Q^{5} T^{6}+34 Q^{3} T^{6}-5 Q^{6} T^{3}+15 Q^{4} T^{3}+15 Q^{4} T^{9}-5 Q^{10} T^{9} \\
& \left.-Q^{7} T^{12}+6 Q^{15} T^{6}+6 Q T^{6}-Q^{2} T^{3}-Q^{2} T^{9}-5 Q^{6} T^{9}\right)\left(Q^{6}-16 Q^{3} T^{3}\right. \\
& +T^{3}-Q^{3}+Q^{4}+Q^{2}-Q^{5}-6 Q^{7} T^{3}-16 Q^{5} T^{3}+Q^{6} T^{6}+Q^{4} T^{6}+Q^{2} T^{6} \\
& \left.+Q^{8} T^{3}-Q^{5} T^{6}-Q^{3} T^{6}+11 Q^{6} T^{3}+22 Q^{4} T^{3}+11 Q^{2} T^{3}-6 T^{3} Q\right)=0 .
\end{aligned}
$$

From [4, Ch. 34, pp.189-190], we note that for $n=1 / 25$ and using the fact that $G_{n}=$ $G_{1 / n}$, we have $G_{1}=1$ and $G_{25}=G_{1 / 25}=\frac{\sqrt{5}+1}{2}$.

Employing the values of $G_{1}$ and $G_{25}$, we can compute the values of $Q$ and $T$, then employing the resulting values of $Q$ and $T$ in (26), we find that the first factor vanishes for a specific value of $q=e^{-\pi / 5}$, thus by the identity theorem it vanishes identically.

Theorem 2 If $\alpha, \beta, \gamma$, and $\delta$ have degrees 1, 5, 5, and 25 respectively, then

$$
\begin{aligned}
& \left(R^{20}+\frac{1}{R^{20}}\right)-14\left(R^{16}+\frac{1}{R^{16}}\right)-29\left(R^{12}+\frac{1}{R^{12}}\right)-144\left(R^{8}+\frac{1}{R^{8}}\right) \\
& -259\left(R^{4}+\frac{1}{R^{4}}\right)-16\left(P^{8}+\frac{1}{P^{8}}\right)\left[\left(R^{8}+\frac{1}{R^{8}}\right)+\left(R^{4}+\frac{1}{R^{4}}\right)+1\right] \\
& +40\left(P^{4}+\frac{1}{P^{4}}\right)\left[\left(R^{12}+\frac{1}{R^{12}}\right)+2\left(R^{8}+\frac{1}{R^{8}}\right)+4\left(R^{4}+\frac{1}{R^{4}}\right)+4\right]=390 .
\end{aligned}
$$

Proof From [3, Entry 13 (xiv), Ch. 19, p. 282], we have

$$
B+\frac{1}{B}+2\left(A-\frac{1}{A}\right)=0,
$$


where $A=\{16 \alpha \beta(1-\alpha)(1-\beta)\}^{1 / 12}$ and $B=\left\{\frac{\beta(1-\beta)}{\alpha(1-\alpha)}\right\}^{1 / 8}$.

Squaring both sides of (28), we get

$$
B^{2}+\frac{1}{B^{2}}+2=\left[2\left(\frac{1}{A}-A\right)\right]^{2} .
$$

Equation (29) can be written as

$$
a+\frac{1}{a}=r-2,
$$

where $a:=\left\{\frac{\beta(1-\beta)}{\alpha(1-\alpha)}\right\}^{1 / 4}$ and $r=\left[2\left(\frac{1}{A}-A\right)\right]^{2}$.

In a similar manner, we find that

$$
D+\frac{1}{D}+2\left(C-\frac{1}{C}\right)=0,
$$

where $C=\{16 \gamma \delta(1-\gamma)(1-\delta)\}^{1 / 12}$ and $D=\left\{\frac{\delta(1-\delta)}{\gamma(1-\gamma)}\right\}^{1 / 8}$.

Equation (31) can be written as

$$
b+\frac{1}{b}=s-2,
$$

where $b:=\left\{\frac{\delta(1-\delta)}{\gamma(1-\gamma)}\right\}^{1 / 4}$ and $s=\left[2\left(\frac{1}{C}-C\right)\right]^{2}$.

Employing (30) and (32) in (25), we get

$$
\begin{aligned}
& 378600 r^{8}+34762089600 r^{4}+1349176960 r^{5} s^{2}+309760 s r^{8}+515545120 s^{6} r \\
& +222121751040 s r^{2}+1333919520 s^{3} r^{4}+1333919520 s^{4} r^{3}-7260 s^{8} r^{3} \\
& +89440 s^{4} r^{7}+73520 r^{6} s^{5}-30 s^{9} r^{4}-90 s^{5} r^{8}-90 s^{8} r^{5}-90 s^{6} r^{7} \\
& -19544320 s^{4} r^{5}+1844560 s^{7} r^{2}+73520 s^{6} r^{5}-90 s^{7} r^{6}+41034107520 s^{4} r \\
& -460 r^{9} s^{2}+41034107520 r^{4} s+76188309120 r^{3} s^{2}+222121751040 s^{2} r \\
& +76188309120 s^{3} r^{2}-19544320 s^{5} r^{4}-23151640 r^{6} s^{3}-30 s^{4} r^{9}-250 s^{9} r^{3} \\
& -842544 s^{7} r^{3}+900 s^{7} r^{5}+2300 s^{8} r^{4}-s^{8} r^{6}+900 s^{5} r^{7}-s^{7} r^{7} \\
& +515545120 s r^{6}+1349176960 s^{5} r^{2}+1844560 s^{2} r^{7}-23151640 s^{6} r^{3} \\
& +2300 s^{4} r^{8}+120982740480 s r+14939378864 s^{4} r^{2}+900 s^{6} r^{6}+20287200 s r^{7} \\
& -115072000 s^{4} r^{4}+138057696000 s r^{3}+138057696000 s^{3} r+18408865664 s^{3} r^{3} \\
& +14939378864 r^{4} s^{2}+78381 r^{6} s^{4}+59398600 r^{6} s^{2}-10710 r^{5} s^{5}+59398600 s^{6} r^{2} \\
& +187184390400 s^{2} r^{2}-s^{6} r^{8}-842544 r^{7} s^{3}+37931 r^{8} s^{2}-250 s^{3} r^{9}-s^{5} r^{9} \\
& +78381 s^{6} r^{4}-135526800 s^{3} r^{5}-135526800 s^{5} r^{3}+6393563744 s^{5} r \\
& +6393563744 r^{5} s+24768320 r^{7}+34762089600 s^{4}+92817446400 s^{3} \\
& +615390640 s^{6}+615390640 r^{6}+378600 s^{8}+6536275200 s^{5}+24768320 s^{7} \\
& +6536275200 r^{5}+92817446400 r^{3}+1160 r^{9}+100818950400 r^{2} \\
& +100818950400 s^{2}+1160 s^{9}-460 s^{9} r^{2}+506 s r^{9}+20287200 s^{7} r+89440 s^{7} r^{4} \\
& -7260 s^{3} r^{8}+37931 s^{8} r^{2}+309760 s^{8} r+506 s^{9} r-s^{9} r^{5}+s^{10}+r^{10}=0 .
\end{aligned}
$$


Substituting for $r, s$ and then setting $A C=P$ and $\frac{A}{C}=R$, we deduce that

$$
\begin{aligned}
& \left(1+80 P^{2} R^{4}+80 P^{6} R^{8}+80 P^{8} R^{6}+160 R^{6} P^{4}+80 P^{4} R^{2}+160 P^{6} R^{4}\right. \\
& -259 P^{6} R^{6}-16 R^{8} P^{4}+R^{1} 0 P^{1} 0-259 P^{4} R^{4}-29 P^{2} R^{2}-16 P^{8} R^{4}-29 P^{8} R^{8} \\
& -16 P^{2} R^{6}-16 P^{6} R^{2}-14 P^{9} R^{9}-16 P^{9} R^{5}+40 P^{9} R^{7}-16 P^{7} R^{3}+160 P^{7} R^{5} \\
& +160 P^{5} R^{7}-144 P^{7} R^{7}-144 P^{3} R^{3}-390 R^{5} P^{5}-16 R^{7} P^{3}-16 P^{5} R^{9}+160 P^{3} R^{5} \\
& \left.+160 P^{5} R^{3}-16 P^{5} R-16 R^{5} P+40 P^{7} R^{9}+40 P^{3} R-14 P R+40 R^{3} P\right) \\
& \left(1+80 P^{2} R^{4}+80 P^{6} R^{8}+80 P^{8} R^{6}+160 R^{6} P^{4}+80 P^{4} R^{2}+160 P^{6} R^{4}\right. \\
& -259 P^{6} R^{6}-16 R^{8} P^{4}+R^{10} P^{10}-259 P^{4} R^{4}-29 P^{2} R^{2}-16 P^{8} R^{4}-29 P^{8} R^{8} \\
& -16 P^{2} R^{6}-16 P^{6} R^{2}+14 P^{9} R^{9}+16 P^{9} R^{5}-40 P^{9} R^{7}+16 P^{7} R^{3}-160 P^{7} R^{5} \\
& -160 P^{5} R^{7}+144 P^{7} R^{7}+144 P^{3} R^{3}+390 R^{5} P^{5}+16 R^{7} P^{3}+16 P^{5} R^{9}-160 P^{3} R^{5} \\
& \left.-160 P^{5} R^{3}+16 P^{5} R+16 R^{5} P-40 P^{7} R^{9}-40 P^{3} R+14 P R-40 R^{3} P\right) \\
& \left(-16 P^{2} R^{4}-16 P^{6} R^{8}-16 P^{8} R^{6}-29 P^{8} R^{2}-29 R^{8} P^{2}-259 R^{6} P^{4}-16 P^{4} R^{2}\right. \\
& -259 P^{6} R^{4}+160 P^{6} R^{6}+80 R^{8} P^{4}+160 P^{4} R^{4}+80 P^{8} R^{4}+80 P^{2} R^{6}+80 P^{6} R^{2} \\
& +P^{10}+R^{10}+40 P^{7} R+40 P R^{7}-14 P^{9} R-16 P^{9} R^{5}-144 P^{7} R^{3}+160 P^{7} R^{5} \\
& +160 P^{5} R^{7}-16 P^{7} R^{7}-16 P^{3} R^{3}-390 R^{5} P^{5}-144 R^{7} P^{3}+40 P^{3} R^{9}-16 P^{5} R^{9} \\
& +160 P^{3} R^{5}+160 P^{5} R^{3}-16 P^{5} R-16 R^{5} P-14 P R^{9}+40 P^{9} R^{3} \text { ) } \\
& \left(-16 P^{2} R^{4}-16 P^{6} R^{8}-16 P^{8} R^{6}-29 P^{8} R^{2}-29 R^{8} P^{2}-259 R^{6} P^{4}-16 P^{4} R^{2}\right. \\
& -259 P^{6} R^{4}+160 P^{6} R^{6}+80 R^{8} P^{4}+160 P^{4} R^{4}+80 P^{8} R^{4}+80 P^{2} R^{6}+80 P^{6} R^{2} \\
& +P^{10}+R^{10}-40 P^{7} R-40 P R^{7}+14 P^{9} R+16 P^{9} R^{5}+144 P^{7} R^{3}-160 P^{7} R^{5} \\
& -160 P^{5} R^{7}+16 P^{7} R^{7}+16 P^{3} R^{3}+390 R^{5} P^{5}+144 R^{7} P^{3}-40 P^{3} R^{9}+16 P^{5} R^{9} \\
& \left.-160 P^{3} R^{5}-160 P^{5} R^{3}+16 P^{5} R+16 R^{5} P+14 P R^{9}-40 P^{9} R^{3}\right)=0 .
\end{aligned}
$$

From [4, Ch. 34, pp.189-190], we note that for $n=1 / 25$ and using the fact that $G_{n}=G_{1 / n}$, we have $G_{1}=1$ and $G_{25}=G_{1 / 25}=\frac{\sqrt{5}+1}{2}$.

Employing the values of $G_{1}$ and $G_{25}$, we can compute the values of $P$ and $R$, and then employing the resulting values of $P$ and $R$ in (34), we find that the third factor vanishes for a specific value of $q=e^{-\pi / 5}$, thus by the identity theorem it vanishes identically.

Theorem 3 If $\alpha, \beta, \gamma$, and $\delta$ have degrees 1, 5, 5, and 25, respectively, then

$$
\begin{aligned}
& \left(T^{20}+\frac{1}{T^{20}}\right)-14\left(T^{16}+\frac{1}{T^{16}}\right)-29\left(T^{12}+\frac{1}{T^{12}}\right)-144\left(T^{8}+\frac{1}{T^{8}}\right) \\
& -259\left(T^{4}+\frac{1}{T^{4}}\right)-16\left(P^{8}+\frac{1}{P^{8}}\right)\left[\left(T^{8}+\frac{1}{T^{8}}\right)+\left(T^{4}+\frac{1}{T^{4}}\right)+1\right] \\
& +40\left(P^{4}+\frac{1}{P^{4}}\right)\left[\left(T^{12}+\frac{1}{T^{12}}\right)+2\left(T^{8}+\frac{1}{T^{8}}\right)+4\left(T^{4}+\frac{1}{T^{4}}\right)+4\right]=390 .
\end{aligned}
$$

Proof Interchanging $\beta$ and $\gamma$ in (27), $P$ remains unchanged whereas $R$ changes to $1 / T$. Hence, we obtain (35). 
Theorem 4 If $\alpha, \beta, \gamma$, and $\delta$ have degrees 1, 5, 4, and 20, respectively, then

$$
\begin{aligned}
& \left(Q^{14}+\frac{1}{Q^{14}}\right)-56\left(Q^{12}+\frac{1}{Q^{12}}\right)+861\left(Q^{10}+\frac{1}{Q^{10}}\right)-5824\left(Q^{8}+\frac{1}{Q^{8}}\right) \\
& +22524\left(Q^{6}+\frac{1}{Q^{6}}\right)-59015\left(Q^{4}+\frac{1}{Q^{4}}\right)+102884\left(Q^{2}+\frac{1}{Q^{2}}\right)-127634 \\
& =\left(T^{12}+\frac{1}{T^{12}}\right)\left[Q^{2}+\frac{1}{Q^{2}}\right]+\left(T^{9}+\frac{1}{T^{9}}\right)\left[16\left(Q+\frac{1}{Q}\right)+3\left(Q^{3}+\frac{1}{Q^{3}}\right)\right. \\
& \left.+8\left(Q^{5}+\frac{1}{Q^{5}}\right)-\left(Q^{7}+\frac{1}{Q^{7}}\right)\right]+\left(T^{6}+\frac{1}{T^{6}}\right)\left[1357\left(Q^{2}+\frac{1}{Q^{2}}\right)\right. \\
& \left.-628\left(Q^{4}+\frac{1}{Q^{4}}\right)+224\left(Q^{6}+\frac{1}{Q^{6}}\right)-24\left(Q^{8}+\frac{1}{Q^{8}}\right)-1196\right] \\
& +\left(T^{3}+\frac{1}{T^{3}}\right)\left[2228\left(Q+\frac{1}{Q}\right)-1800\left(Q^{3}+\frac{1}{Q^{3}}\right)+1261\left(Q^{5}+\frac{1}{Q^{5}}\right)\right. \\
& \left.-336\left(Q^{7}+\frac{1}{Q^{7}}\right)+109\left(Q^{9}+\frac{1}{Q^{9}}\right)-16\left(Q^{11}+\frac{1}{Q^{11}}\right)\right] .
\end{aligned}
$$

Proof Replacing $q$ by $q^{4}$ in (20), $m$ and $a$ changes to $m^{\prime}$ and $b$, respectively, then we arrive at

$$
m^{\prime}+b=\frac{5 b}{m^{\prime}}+1
$$

where $m^{\prime}:=\frac{\varphi^{2}\left(q^{4}\right)}{\varphi^{2}\left(q^{20}\right)}$ and $b:=\left\{\frac{\delta(1-\delta)}{\gamma(1-\gamma)}\right\}^{1 / 4}$.

Employing (20) and (37) in the (11), we obtain (36). Since the proof of (36) runs on the same line as the proof of Theorem 1 , so we omit the details.

Theorem 5 If $\alpha, \beta, \gamma$, and $\delta$ have degrees 1, 5, 4, and 20, respectively, then

$$
\begin{aligned}
& P^{32}-3072 P^{24}-444544 P^{16}-\left(7032832 P^{8}+\frac{26738688}{P^{8}}\right) \\
& +2^{7}\left(R^{4}+\frac{1}{R^{4}}\right)\left[2^{6}\left(1961 P^{4}+\frac{4416}{P^{4}}\right)+24\left(595 P^{12}+\frac{2048}{P^{12}}\right)+355 P^{20}\right] \\
& -2^{8}\left(R^{8}+\frac{1}{R^{8}}\right)\left[\left(16433 P^{8}+\frac{65536}{P^{8}}\right)+8\left(111 P^{16}+\frac{512}{P^{16}}\right)+84352\right] \\
& +2^{9}\left(R^{12}+\frac{1}{R^{12}}\right)\left[\left(1033 P^{12}+\frac{8192}{P^{12}}\right)+224\left(52 P^{4}+\frac{135}{P^{4}}\right)\right] \\
& -2^{14}\left(R^{16}+\frac{1}{16}\right)\left[3\left(13 P^{8}+\frac{96}{P^{8}}\right)+290\right]+2^{14}\left(R^{20}+\frac{1}{R^{20}}\right) \\
& {\left[25 P^{4}+\frac{96}{P^{4}}\right]-2^{17}\left(R^{24}+\frac{1}{R^{24}}\right)+\frac{2^{14}}{P^{4}}\left(R^{28}+\frac{1}{R^{28}}\right)=33554432 .}
\end{aligned}
$$

Proof The proof of (38) is similar to the proof of (27). Hence, we omit the details. 
Theorem 6 If $\alpha, \beta, \gamma$, and $\delta$ have degrees 1, 5, 4, and 20, respectively, then

$$
\begin{aligned}
& P^{32}-3072 P^{24}-444544 P^{16}-\left(7032832 P^{8}+\frac{26738688}{P^{8}}\right) \\
& +2^{7}\left(T^{4}+\frac{1}{T^{4}}\right)\left[2^{6}\left(1961 P^{4}+\frac{4416}{P^{4}}\right)+24\left(595 P^{12}+\frac{2048}{P^{12}}\right)+355 P^{20}\right] \\
& -2^{8}\left(T^{8}+\frac{1}{T^{8}}\right)\left[\left(16433 P^{8}+\frac{65536}{P^{8}}\right)+8\left(111 P^{16}+\frac{512}{P^{16}}\right)+84352\right] \\
& +2^{9}\left(T^{12}+\frac{1}{T^{12}}\right)\left[\left(1033 P^{12}+\frac{8192}{P^{12}}\right)+224\left(52 P^{4}+\frac{135}{P^{4}}\right)\right] \\
& -2^{14}\left(T^{16}+\frac{1}{16}\right)\left[3\left(13 P^{8}+\frac{96}{P^{8}}\right)+290\right]+2^{14}\left(T^{20}+\frac{1}{T^{20}}\right) \\
& {\left[25 P^{4}+\frac{96}{P^{4}}\right]-2^{17}\left(T^{24}+\frac{1}{T^{24}}\right)+\frac{2^{14}}{P^{4}}\left(T^{28}+\frac{1}{T^{28}}\right)=33554432 .}
\end{aligned}
$$

Proof Interchanging $\beta$ and $\gamma$ in (38), $P$ remains unchanged whereas $R$ changes to $1 / T$. Hence, we obtain (39).

Theorem 7 If $\alpha, \beta, \gamma$, and $\delta$ have degrees 1, 5, 11, and 55, respectively, then

$$
\begin{aligned}
& {\left[T^{5}+\frac{1}{T^{5}}\right]+11\left\{3\left[T+\frac{1}{T}\right]+8\left[T^{2}+\frac{1}{T^{2}}\right]+\left[T^{3}+\frac{1}{T^{3}}\right]-\left[T^{4}+\frac{1}{T^{4}}\right]-15\right\}} \\
& =\left[Q^{6}+\frac{1}{Q^{6}}\right]-\left[Q^{3}+\frac{1}{Q^{3}}\right]\left\{22\left[T+\frac{1}{T}\right]+11\left[T^{2}+\frac{1}{T^{2}}\right]+33\right\} .
\end{aligned}
$$

Proof Replacing $q$ by $q^{11}$ in equation (20), $m$ and $a$ changes to $m^{\prime}$ and $b$, respectively, then we arrive at

$$
m^{\prime}+b=\frac{5 b}{m^{\prime}}+1 \text {, }
$$

where $m^{\prime}:=\frac{\varphi^{2}\left(q^{11}\right)}{\varphi^{2}\left(q^{55}\right)}$ and $b:=\left\{\frac{\delta(1-\delta)}{\gamma(1-\gamma)}\right\}^{1 / 4}$.

Employing (20) and (41) in (13), we obtain (40). Since the proof of (40) runs on the same line as the proof of Theorem 1, so we omit the details.

Theorem 8 If $\alpha, \beta, \gamma$, and $\delta$ have degrees 1, 5, 11, and 55, respectively, then

$$
\begin{aligned}
& \left(R^{24}+\frac{1}{R^{24}}\right)+176\left(R^{20}+\frac{1}{R^{20}}\right)+3872\left(R^{16}+\frac{1}{R^{16}}\right)+25476\left(R^{12}+\frac{1}{R^{12}}\right) \\
& +181984\left(R^{8}+\frac{1}{R^{8}}\right)+472560\left(R^{4}+\frac{1}{R^{4}}\right)+1295558-1024\left(P^{20}+\frac{1}{P^{20}}\right) \\
& +\left(P^{16}+\frac{1}{P^{16}}\right)\left[5632\left(R^{4}+\frac{1}{R^{4}}\right)+25344\right]-\left(P^{12}+\frac{1}{P^{12}}\right)\left[53504\left(R^{4}+\frac{1}{R^{4}}\right)\right. \\
& \left.+10560\left(R^{8}+\frac{1}{R^{8}}\right)+138688\right]+\left(P^{8}+\frac{1}{P^{8}}\right)\left[217536\left(R^{4}+\frac{1}{R^{4}}\right)+45584\right. \\
& \left.\times\left(R^{8}+\frac{1}{R^{8}}\right)+8096\left(R^{12}+\frac{1}{R^{12}}\right)+430496\right]-\left(P^{4}+\frac{1}{P^{4}}\right)\left[406736\left(R^{4}+\frac{1}{R^{4}}\right)\right. \\
& \left.+126280\left(R^{8}+\frac{1}{R^{8}}\right)+19888\left(R^{12}+\frac{1}{R^{12}}\right)+2376\left(R^{16}+\frac{1}{R^{16}}\right)+962016\right]=0 .
\end{aligned}
$$


Proof The proof of (42) is similar to the proof of (27). Hence, we omit the details.

Theorem 9 If $\alpha, \beta, \gamma$, and $\delta$ have degrees $1,5,11$, and 55, respectively, then

$$
\begin{aligned}
& \left(T^{24}+\frac{1}{T^{24}}\right)+176\left(T^{20}+\frac{1}{T^{20}}\right)+3872\left(T^{16}+\frac{1}{T^{16}}\right)+25476\left(T^{12}+\frac{1}{T^{12}}\right) \\
& +181984\left(T^{8}+\frac{1}{T^{8}}\right)+472560\left(T^{4}+\frac{1}{T^{4}}\right)+1295558-1024\left(P^{20}+\frac{1}{P^{20}}\right) \\
& +\left(P^{16}+\frac{1}{P^{16}}\right)\left[5632\left(T^{4}+\frac{1}{T^{4}}\right)+25344\right]-\left(P^{12}+\frac{1}{P^{12}}\right)\left[53504\left(T^{4}+\frac{1}{T^{4}}\right)\right. \\
& \left.+10560\left(T^{8}+\frac{1}{T^{8}}\right)+138688\right]+\left(P^{8}+\frac{1}{P^{8}}\right)\left[217536\left(T^{4}+\frac{1}{T^{4}}\right)+45584\right. \\
& \left.\times\left(T^{8}+\frac{1}{T^{8}}\right)+8096\left(T^{12}+\frac{1}{T^{12}}\right)+430496\right]-\left(P^{4}+\frac{1}{P^{4}}\right)\left[406736\left(T^{4}+\frac{1}{T^{4}}\right)\right. \\
& \left.+126280\left(T^{8}+\frac{1}{T^{8}}\right)+19888\left(T^{12}+\frac{1}{T^{12}}\right)+2376\left(T^{16}+\frac{1}{T^{16}}\right)+962016\right]=0 .
\end{aligned}
$$

Proof Interchanging $\beta$ and $\gamma$ in (42), $P$ remains unchanged whereas $R$ changes to $1 / T$. Hence, we obtain (9).

Acknowledgments The authors are grateful to the referee for their valuable remarks and suggestions which considerably improved the quality of the paper. The first author is thankful to Department of Science and Technology (DST), New Delhi, India for support under the research project SR/S4/MS:739/11.

\section{References}

1. Baruah, N.D.: A few theta-function identities and some of Ramanujan's modular equations. Ramanujan J. 4, 239-250 (2000)

2. Baruah, N.D.: On some of Ramanujan's Schläfli-type "mixed" modular equations. J. Number Theory 100, 270-294 (2003)

3. Berndt, B.C.: Ramanujan's Notebooks, Part III. Springer, New York (1991)

4. Berndt, B.C.: Ramanujan's Notebooks, Part V. Springer, New York (1998)

5. Kim, T., Mahadeva Naika, M.S., Chandankumar, S., Jang, L.-C., Kim, Y.-H., Lee, B.: On some new Schläfli-type cubic modular equations. Adv. Stud. Contemp. Math. 20, 63-80 (2010)

6. Mahadeva Naika, M.S.: Some new explicit values for Ramanujan's class invariants. Adv. Stud. Contemp. Math. 20, 557-568 (2010)

7. Mahadeva Naika, M.S., Chandankumar, S.: Some new Schläfli-type modular equations in the quartic theory. Ramanujan Rediscov 14, 185-199 (2010)

8. Mahadeva Naika, M.S., Dharmendra, B.N., Chandankumar, S.: New modular relations for Ramanujan's parameter $\mu(q)$. Int. J. Pure Appl. Math. 74, 413-435 (2012)

9. Mahadeva Naika, M.S., Suman, N.P., Chandankumar, S.: Schläfli-type mixed modular equations of degrees $1,3, n$ and $3 n$. Preprint

10. Mahadeva Naika, M.S., Sushan Bairy, K.: On some new explicit evaluations of class invariants. Vietnam J. Math. 36, 103-124 (2008)

11. Mahadeva Naika, M.S., Sushan Bairy, K.: On some new Schläfli-type mixed modular equations. Adv. Stud. Contemp. Math. 21, 189-206 (2011)

12. Mahadeva Naika, M.S., Sushan Bairy, K.: Some modular equations in the form of Schläfli. Ital. J. Pure Appl. Math. 30, 233-252 (2013)

13. Ramanathan, K.G.: Ramanujan's modular equations. Acta Arith. 53, 403-420 (1989)

14. Ramanujan, S.: Notebooks, Vols. 1 and 2. Tata Institute of Fundamental Research, Bombay (1957)

15. Russell, R.: On $k \lambda-k^{\prime} \lambda^{\prime}$ modular equations. Proc. Lond. Math. Soc. 19, 90-111 (1887)

16. Schläfli, L.: Beweis der Hermiteschen Verwandlungstafeln für die elliptischen Modularfunctionnen. J. Reine Angew. Math. 72, 360-369 (1870)

17. Vasuki, K.R., Sreeramurthy, T.G.: Certain new Ramanujan's Schläfli type mixed modular equations. J. Math. Anal. Appl. 309, 238-255 (2005)

18. Weber, H.: Zur theorie der elliptischen functionen. Acta Math. 11, 333-390 (1887) 